\title{
Large time-varying inductance load for studying power flow on the $Z$ machine
}

\author{
Andrew Porwitzky $\odot,{ }^{1, *}$ Brian T. Hutsel, ${ }^{1}$ Christopher T. Seagle, ${ }^{1}$ Tommy Ao, ${ }^{1}$ Sean Grant, ${ }^{1}$ \\ Aaron Bernstein, ${ }^{2}$ Jung-Fu Lin, ${ }^{3}$ and Todd Ditmire ${ }^{4}$ \\ ${ }^{1}$ Sandia National Laboratories, PO Box 5800 Albuquerque, New Mexico 87185, USA \\ ${ }^{2}$ University of Texas at Austin, 2515 Speedway, Austin, Texas 78712, USA \\ ${ }^{3}$ University of Texas at Austin, 2305 Speedway Stop C1160, Austin, Texas 78712-1692, USA \\ ${ }^{4}$ University of Texas at Austin, C1600 Austin, Texas 78712, USA
}

(Received 29 April 2019; published 4 September 2019)

\begin{abstract}
Interest in studying power flow dynamics has grown in recent years, with new power flow diagnostics being developed at Sandia National Laboratories for the $Z$ Pulsed Power Facility. Presently, the only power flow loads that have been studied are cylindrical static or imploding loads that are driven by synchronous short pulse (100 ns rise time). Presented is a design that utilizes the dynamic materials properties program's stripline geometry in a high voltage pulsed shaped (asymmetric asynchronous) driving mode. This design has exhibited repeatable current loss with a large time-varying inductance that is well matched to the machine at pulse initialization but which triples to high inductance in $800 \mathrm{~ns}$. Evidence is presented that plasma not captured in the magnetohydrodynamic approximation and ill represented by any of our existing predictive pulsed power codes is adversely affecting load current delivery. The authors believe this design could be of great interest to the experimental and modeling communities for studying power flow dynamics.
\end{abstract}

DOI: 10.1103/PhysRevAccelBeams.22.090401

\section{INTRODUCTION}

Interest in studying loss mechanisms and overall power flow dynamics on large pulsed power drivers has grown in recent years, with new power flow diagnostics being developed at Sandia National Laboratories for the $Z$ Pulsed Power Facility, commonly known as the $Z$ machine [1-5]. Presently, the only power flow loads that have been studied are cylindrical static or imploding loads that are driven by synchronous short pulse (100 ns rise time). This is a logical decision for early phase studies for a number of reasons: (1) The most significant and repeatable current loss is encountered in short pulse experiments; (2) most short pulse experiments have comparable load inductance time histories, allowing for the development of empirical predictive loss models; (3) synchronous pulse experiments impose a simplifying azimuthal symmetry across the entire driver; (4) synchronous short pulse experiments are most desirable in inertial confinement fusion experiments where current loss can prevent access to critical regimes both on existing and proposed future drivers. Though it makes

\footnotetext{
*ajporwi@sandia.gov
}

Published by the American Physical Society under the terms of the Creative Commons Attribution 4.0 International license. Further distribution of this work must maintain attribution to the author(s) and the published article's title, journal citation, and DOI. perfect sense to study synchronous short pulse power flow initially (and into the future as diagnostic and modeling techniques mature) there is great value in testing emerging power flow models on targets and current pulse shapes that exist well outside the narrow band of cylindrically imploding synchronous short pulse experiments.

There are two main characteristics that a load must have in order to be of practical interest as a power flow diagnostic platform. First, the target must be well understood and easy to manufacture and install. The dynamic materials program (DMP) stripline geometry has been used in its present form for nearly a decade on the $Z$ machine [6-11]. The DMP stripline, illustrated in Fig. 1, resembles a horseshoe with the anode stripline attached to the cathode stripline via a small rectangular short at the top of the load. As current flows on the inner surface of the stripline a strong (routinely 900 Tesla) magnetic field is developed in the $1 \mathrm{~mm}$ load anode-cathode (AK) gap. This magnetic field begins blowing the stripline apart, causing samples attached on the outside of the panels (not shown in Fig. 1) to undergo controlled compression to pressures of 100s of GPa. Velocimetry probes placed in magnetically shielded housings attached to the outside of the stripline measure the velocity response of the sample materials under high compression in order to infer equation of state information [8-11]. Figure 2 shows a photograph of $Z$ machine experiment Z3260 which employed the specific load we will discuss here. Visible are the magnetic shielding 


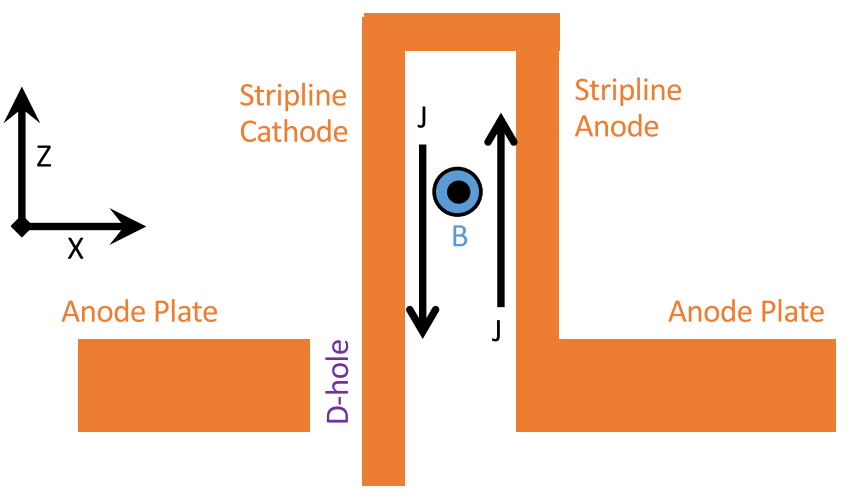

FIG. 1. Illustration of stripline load geometry. The stripline is mounted directly to the $Z$ machine top plate on the anode side, while the cathode stalk passes through a D-shaped hole (the flat side of the hole is flush with the anode stalk) in order to connect to the upper cathode (not shown). The D-hole geometry allows for a break from the standard cylindrical power flow geometry of the $Z$ machine.

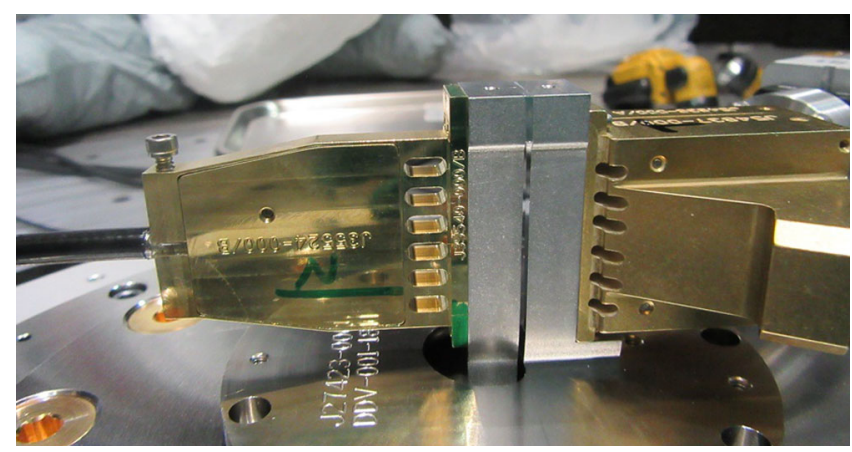

FIG. 2. Photograph of stripline load geometry installed in the $Z$ machine prior to current discharge on experiment Z3260. The cathode stripline is on the left and can be seen passing through the D-hole. Magnetically shielded mounts housing the velocimetry probes are shown attached to both cathode and anode striplines.

mounts that house the velocimetry probes [12-14]. With nearly a decade of successful experiments, the stripline geometry is well understood, with magnetohydrodynamic (MHD) simulations routinely producing accurate predictions of observed stripline velocities.

The second requirement is that the target must have a repeatable and predicable loss characteristic. This has historically not been easy to achieve on a DMP target, as most $Z$ machine DMP targets have minimal loss that can vary on repeat shots. It is notoriously difficult to predict loss on DMP experiments because (1) loss mechanisms are not presently well understood, and (2) almost every DMP current pulse shape is substantially different from preceding pulse shapes. The reason for vastly different pulse shapes is due to the experimental objectives driving the design, but generally DMP pulse shapes vary in rise time from 300-1500 ns and have widely varying $d I / d t$ within that time. In the section immediately following we will describe how the proposed power flow load design was driven by unique and extreme experimental equation of state measurement requirements.

We aim to present a case for the use of the proposed load geometry and pulse shape as a future benchmark test case for modeling the asynchronous asymmetric pulse shaped class of experiments. We believe that this experiment can be used to rigorously exercise future predictive pulsed power loss codes, providing shake-down testing of theoretical models.

In Sec. II we provide details on the load geometry and pulse shape. Section III reviews what our existing loss mechanism models are able to predict concerning this configuration. Section IV provides evidence for the case of plasma affecting power flow in the region of the load. Finally, we end with conclusions and a call for collaboration.

\section{EXPERIMENTAL SPECIFICATIONS AND MOTIVATING DESIGN CRITERIA}

The original purpose of the stripline experiment we now propose to use as a power flow target was to gather equation of state information for iron at Earth's core conditions as part of the $Z$ Fundamental Science Program. For clarity we will refer to this load geometry and pulse shape combination as PF. Design objectives for the PF experiment called for launching an aluminum flyer at an iron sample such that a $300 \mathrm{GPa}$ shock pressure was established in the iron, thus shock-melting the iron into a liquid state [15]. This necessitated an aluminum impact velocity of $11 \mathrm{~km} / \mathrm{s}$. The current pulse was designed to hold pressure constant on the sample after flyer impact to support the propagation of a steady shock through the iron before the current ramped up, isentropically compressing the iron sample to a peak pressure of at least $360 \mathrm{GPa}$ in order to match or exceed the pressure conditions at the Earth's core. Any pressure achieved in excess of $360 \mathrm{GPa}$ would extend the relevant equation of state analysis to the iron cores of super-Earth planets, which was of course desirable [16,17]. Though the design criteria conceptually match what is typically performed in a shock-ramp experiment, the current hold to establish constant shock pressure must occur at a very high magnitude in order to support a $300 \mathrm{GPa}$ shock. As a result of such a high current hold, the overall pulse shape had to be made short compared to typical shock-ramp pulses [18] in order to maximize use of the stored $Z$ machine energy. The resulting current pulse thus has a very high $d I / d t$ compared to most shock-ramp pulses. Figure 3 shows the PF current pulse produced by the $Z$ circuit model [19] and the associated time-varying load inductance; a reference DMP shock-ramp pulse is included for comparison. The load inductance in the stripline geometry is driven by the divergence of the anode and cathode striplines as the load AK gap opens under magnetic pressure. The load inductance is extracted from two-dimensional MHD simulations which track the opening of this AK gap as well as the total 


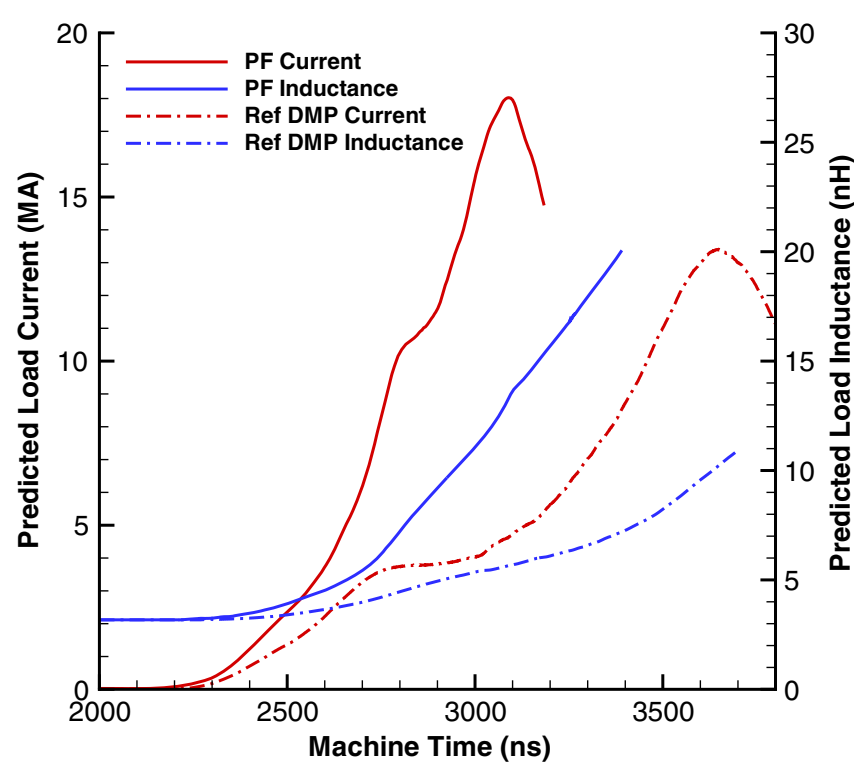

FIG. 3. Plot of predicted current and associated load inductance for the PF design compared to a reference DMP design.

magnetic energy, $\varepsilon$. The inductance, $L$, is then calculated from the fundamental relation [20] $L=2 \varepsilon / I^{2}$. Timevarying geometric divergence, magnetic diffusion, and material vaporization effects are captured by the multiphysics MHD code in the calculation of $\varepsilon$. The MHD code used here is Alegra, which has been benchmarked across many DMP experiments [21].

The PF configuration used the standard $11 \mathrm{~mm}$ wide, three-sample pair stripline load. At each location the AL6061 panel floor (the flyer) was $2.8 \mathrm{~mm}$ thick with a 600 micron flight gap built into the panel. Samples of 0.9$1.2 \mathrm{~mm}$ thick iron backed by $2.5 \mathrm{~mm}$ thick lithium fluoride windows were impacted by the flyer. It is worth noting that none of these materials or procedures are atypical of a $Z$ machine DMP stripline load. One of the sample locations on Z3260 left out the iron sample and window, permitting a velocimetry-based load current unfold, allowing for direct inference of current loss [22].

\section{DIAGNOSIS OF LOSS MECHANISM}

We will use the standard current inference B-dots [23] located in the magnetically insulated transmission lines (MITLs) as well as load current velocimetry [22] to diagnose current loss. The MITL B-dots are positioned at a radius of $66 \mathrm{~cm}$ from the center of the $Z$ machine where loss is not typically observed on DMP targets, meaning the Z Circuit Model accurately predicts the pulse shape at this location. Thus we expect the inferred MITL current to match our predicted load current to within $Z$ machine variations and B-dot accuracy $( \pm 5 \%)$, and we assert that this is the case given the comparison shown in Fig. 4. The purpose of the MITL comparison is to demonstrate that the $Z$ machine attempted to deliver the

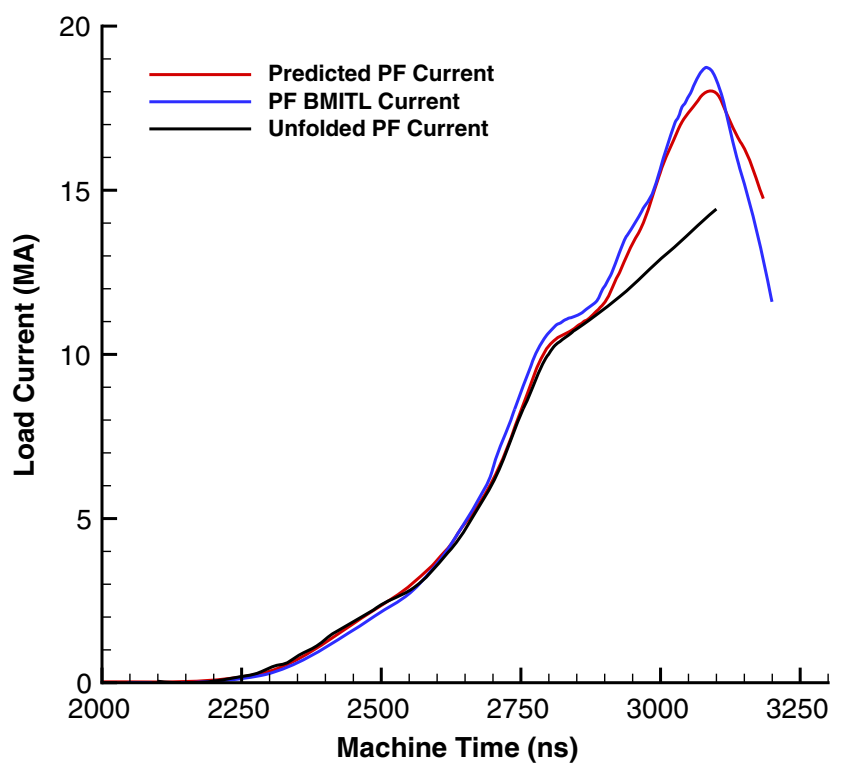

FIG. 4. Plot of predicted current for the PF target along with the experimental current measured by the MITL B-dots and the best attempt at unfolding the load current from target velocimetry using experimental data obtained from Z3260.

desired current pulse to the load, and we see from the late time behavior that the pulse shape was achievable as designed at the MITL B-dot radius.

Load current velocimetry clearly indicates that a significant loss mechanism kicked in around $2900 \mathrm{~ns}$ when $d I / d t$ was increasing to ramp the iron sample after the shock pressure hold (the stable region of the current that begins at $2750 \mathrm{~ns}$ ). The first use of the PF configuration was Z3155, though a velocimetry measurement of the complete drive was not taken due to all locations on the stripline containing samples. Witness windows above and below the samples allow for velocimetry measurements of the flyer up to the time of impact. Z3260 was an exact repeat of Z3155 but with one sample omitted to allow a full drive measurement. Drive measurements from Z3155 and Z3260 match drive predictions until just after the $d I / d t$ drop associated with the beginning of the shock pressure hold at $2750 \mathrm{~ns}$, at which time impact occurs. Sample velocities from Z3155 and Z3260 indicate that both experiments underwent the same loading beginning at $2750 \mathrm{~ns}$, which implies that the loss mechanism is repeatable despite not having independent drive measurements on both experiments. Due to the uncertain nature of the iron equation of state in this area of phase space a reliable load current unfold can not be performed using iron sample velocities. The unfold method will be discussed further in Sec. IV.

One potential theory for the loss mechanism comes from the flow current model outlined by Hutsel et al. in Ref. [19]. The electron flow current traveling in a magnetically insulated layer above each cathode MITL is represented in the $Z$ circuit model as 


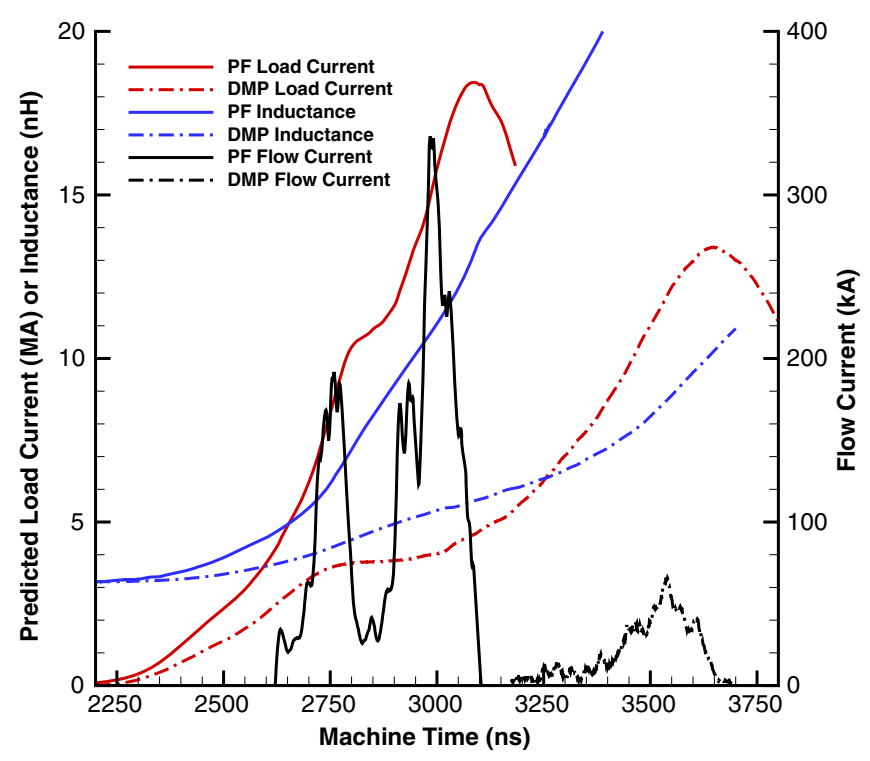

FIG. 5. Load current (red), inductance (blue), and $Z$ circuit model flow current (black) for the PF experiment (solid) and a reference DMP experiment (dashed). The flow current shown has been summed from each of the four MITLs. Peak flow current in the near lossless DMP case is $60 \mathrm{kA}$, less than one fifth of the peak flow current of the PF design.

$$
I_{f}=\frac{13}{16} \frac{V^{2}}{I_{a} Z^{2}}
$$

where $V$ is the MITL voltage, $I_{a}$ is the current flowing through the metallic anode, and $Z$ is the vacuum impedance of the MITL region. Current is split on the cathode side between that flowing through the metallic cathode, $I_{k}$, and the flow current such that $I_{a}=I_{k}+I_{f}$. The $Z$ circuit model calculates flow current at each of the four MITL levels prior to their merger at the post-hole convolute. If this flow current were to lose insulation at the convolute and stream into the load it could represent a plasma source that could partially short the load. We sum the flow current from all four levels and plot the result on the same time axis as the predicted current and inductance in Fig. 5. For comparison we have included the flow current from the reference DMP shot, which exhibited very little load current loss. Note that these values all come directly from predictive simulations, and do not purport to match the behavior of the target after the loss mechanism triggers.

The flow current model is simple, and is provided as one possible source of loss in the form of an electron plasma that could short the feed or load. This behavior is not included in the treatment of the flow current in the $Z$ circuit model, and represents an effect that would have to be modeled in a multiphysics target dynamics code. We propose that the electron flow current, which is $\mathrm{kA}$ in magnitude, can be responsible for MA of current shorting at the target if this electron plasma partially closes the $1 \mathrm{~mm}$ AK gap in the stripline geometry.
Though the PF configuration has higher flow current than the reference DMP configuration, the timing of the onset of the flow current does not correlate to the observed loss mechanism. The flow current spikes as $d I / d t$ climbs toward the shock pressure hold, however there is no noticeable loss until the end of the shock hold in the experimental data. We would expect to see a significant spike in flow current at that time, however the corresponding spike is slightly smaller than the initial spike. Quantitatively, the flow current model does not well match our loss observations.

The flow current plays a roll in an additional loss mechanism that is not included in the $Z$ circuit model utilized above, but is described in Ref. [19], namely the positive ion current. Ion generation is sourced under the assumption that the MITLs behave as an enhanced space charge limited (SCL) emission source, such that the ion current is given by

$$
I_{\mathrm{SCL}}=\frac{4 \epsilon_{o}}{9} V^{3 / 2} \sqrt{2 \gamma} \frac{A}{h^{2}},
$$

where $\epsilon_{o}$ is the permittivity of free space, $h$ is the AK gap spacing at that location of the power flow, $A$ is the area of the computational element in the model, $\gamma$ is the ion charge to mass ratio, and $V$ is the electric potential in the AK gap. Negative space charge can accumulate in the AK gap due to the presence of the electron flow current; such negative space charge enhances the ion emission. We can define an enhancement parameter $\eta$ such that

$$
\eta=\frac{3 h}{4 \epsilon_{o} A V} k Q_{e}, \quad \eta \geq 1
$$

where the inequality is forced to ensure that the electron flow current never decreases the ion emission. The new variables in Eq. (3) represent the accumulated electron charge $\left(Q_{e}\right)$ and a nondimensional constant representing the average ion velocity in the AK gap $(k)$ [19]. We now define the enhanced space charge limited current as

$$
I_{\mathrm{ion}}=\eta I_{\mathrm{SCL}} .
$$

We can see from Eq. (3) that the more electron flow current that accumulates $\left(Q_{e}\right)$ the higher the ion loss current $\left(I_{\text {ion }}\right)$. Additionally, the voltage at any location is dependent on the current at that location as well as the total inductance of the up-stream components such that

$$
V=I_{a} \frac{d L}{d t}+L \frac{d I_{a}}{d t} .
$$

Examining Eqs. (1)-(4) we find that $I_{\text {ion }} \propto \sqrt{V}$, so we expect high inductance, high current loads to experience both more electron flow current (which scales as $V^{2}$ ) and ion loss current.

The result of an ion loss current calculation for the PF target is shown in Fig. 6. The ion loss current is subtracted from the $Z$ circuit model prediction (which already has the 


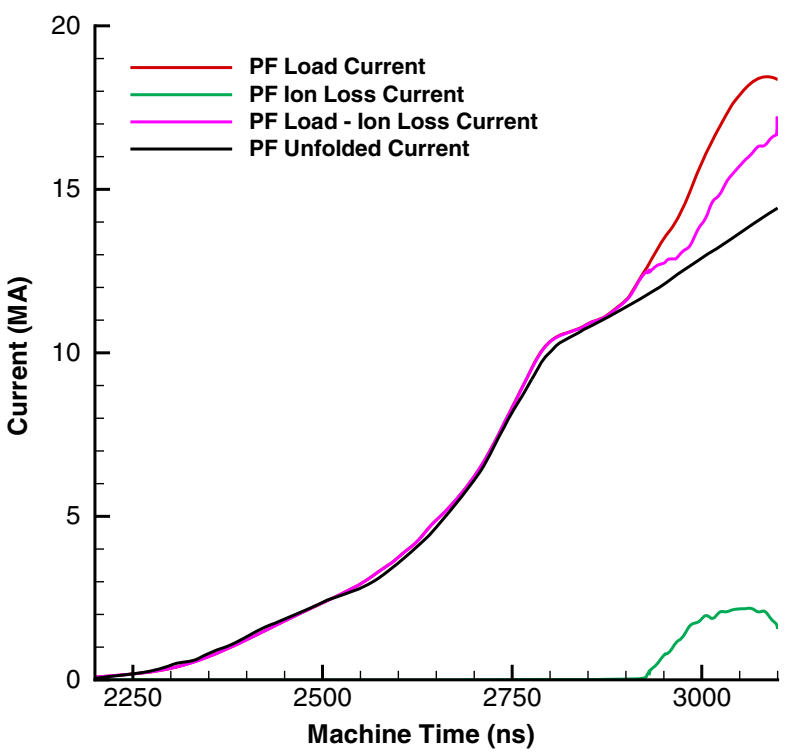

FIG. 6. Ion loss current (green), $Z$ circuit model load current prediction (red), and the velocimetry-based load current unfold (black) compared to the circuit model prediction less the ion loss current (pink). Though the ion loss does not completely account for the estimated load current, it does indicate that catastrophic loss could be qualitatively predicted.

electron flow current loss taken into account) and compared to the unconverged velocimetry-based load current unfold. We see that the ion loss current, which peaks at approximately $2 \mathrm{MA}$, is insufficient to account for the magnitude of the current loss, though it does predict that significant current loss would take place and at approximately the correct time. As stated above, the ion current loss is triggered by the existence of electron flow current, which from Fig. 5 is 200-300 kA for most of the lossy regions of the pulse. This yields an $\eta \approx 10$. For a sense of scale, MA of flow current corresponds to $\eta \approx 30$. Further estimates and bounds for $\eta$ can be found in Appendix C of Ref. [19].

Present theories for current loss in the load region state that undesirable plasma generation results in some level of current shorting at or within the convolute radius. Possible plasma sources being considered include the aforementioned electron flow current, enhanced SCL ion emission, desorbed and ionized water/contaminants from the MITL surfaces, and/or vaporized metal due to heavy Joule heating $[3,19,24]$. Beyond the classical shorting that can occur due to these plasmas, in the next section we will present evidence for the presence of plasma invalidating the fundamental MHD assumptions used to model $Z$ machine target dynamics.

\section{EVIDENCE FOR PRESENCE OF LOAD PLASMA}

Circumstantial evidence for the presence of load plasma appeared during the unfold procedure to determine the load

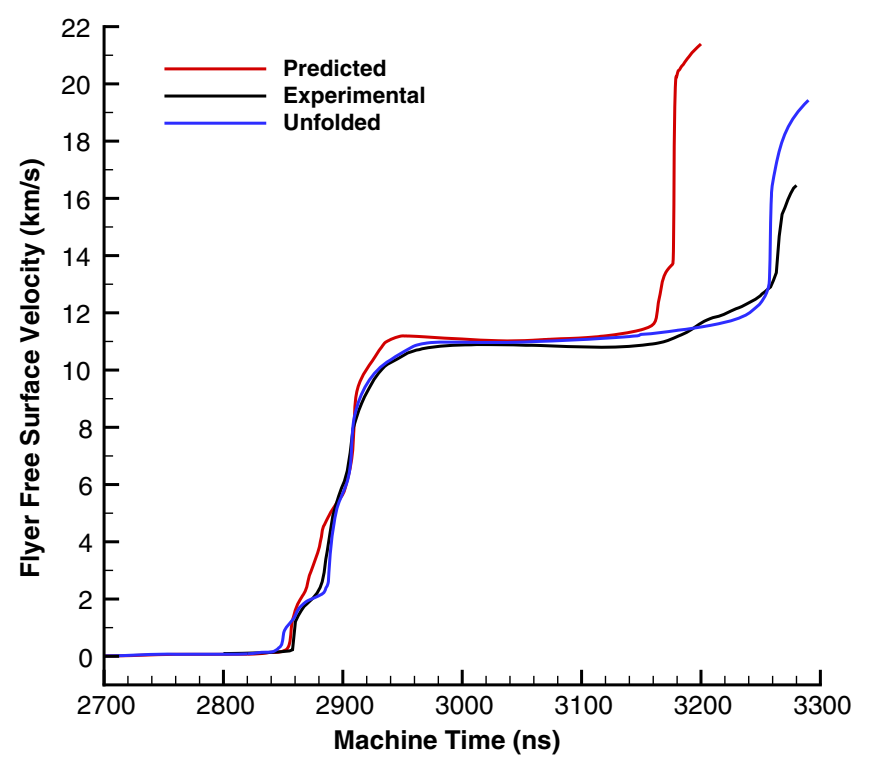

FIG. 7. Relevant velocities for the load current unfold for Z3260. The predicted flyer free surface velocity is shown in red. The experimental velocity (black) indicates significant loss after the shock hold. The velocity resulting from the best solution unfold is shown in blue.

current from Z3260 velocimetry. The previously mentioned unfold procedure is detailed elsewhere [22], and it is sufficient to say here that the experimentally measured flyer free surface velocity is used as a target metric to adjust the magnetic field drive used in a one-dimensional MHD simulation to reproduce the experimental velocity; thus the magnetic drive is "unfolded" from the observed velocity profile. In standard unfolds-especially those pertaining to DMP experiments-the maximum global residual observed for isentropically compressed materials is typically $20-40 \mathrm{~m} / \mathrm{s}$. Figure 7 shows the predicted flyer free surface velocity which neglects all loss mechanisms, along with the experimentally measured flyer velocity. The best solution velocity returned by the unfold framework is also shown in Fig. 7, and we can clearly see that the method failed to converge though it got qualitatively close. Note that for the drive unfold only the material models for the standard stripline aluminum (AL6061) are needed, and those models are heavily validated and facilitate convergence to low residual $(20-40 \mathrm{~m} / \mathrm{s})$ solutions [22]. The unfold from the PF experiment is poor to an unprecedented degree. The unfold framework employed here typically converges without trouble, so this represents an anomaly.

Porwitzky et al. [5] present evidence that low density plasma—order 1e16 electron/cc — can affect power flow in ways detectable using load current velocimetry. Plasmas of that magnitude are below the modeling threshold of the MHD assumption and thus key load physics is not being accurately captured. In previous work the effect of this low density plasma was an order $1 \%$ inaccuracy in load current inference. The appearance of low density plasma also 


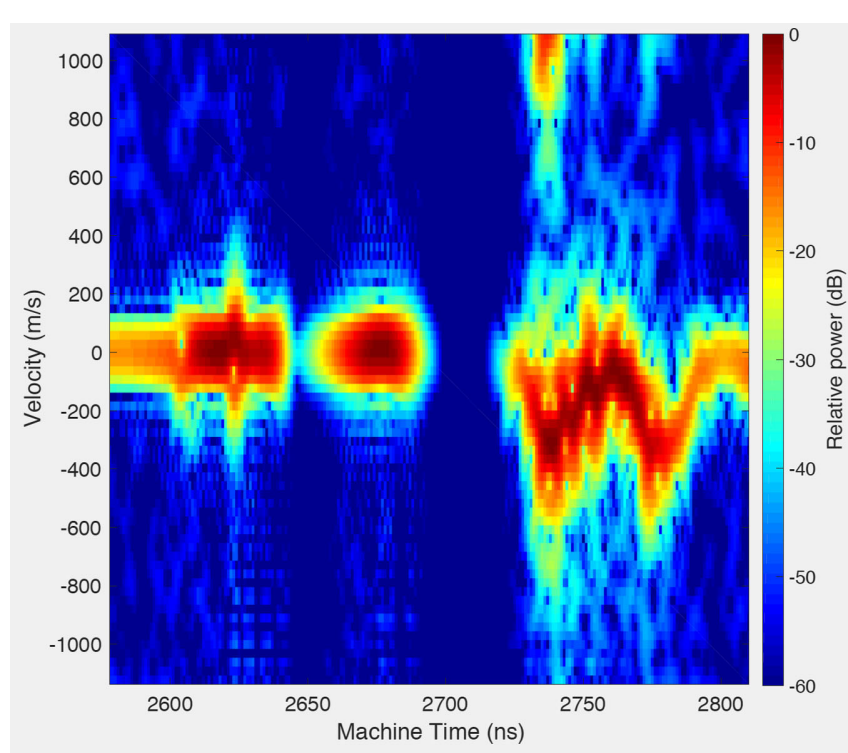

FIG. 8. PDV spectrogram from the D-hole plasma detector deployed on Z3339. First plasma signal occurs at $2600 \mathrm{~ns}$, followed by intermittent signal and complete signal recovery at 2720 ns.

temporally correlated with standard current loss onset. What we have here is a catastrophic current loss mechanism much more severe than any analyzed in Ref. [5]. We propose that the loss mechanism triggered by the steep inductance rise of our target is caused by the generation of plasma, most likely caused by a bad impedance match to the generator, which then flows up the MITL feed and along the load AK gap. The presence of such a plasma, which is not sourced in the MHD model due to our inability to predict its exact character and origin, would alter current coupling to the stripline in ways that the existing MHD models cannot capture. The plasma properties could be so extreme that an unfold solution does not exist in the framework of MHD absent an accurate source for the loss plasma.

A photonic Doppler velocimetry-based plasma diagnostic was deployed across the D-hole in order to attempt to verify the presence of plasma exiting the feed. The plasma detector exploits the principles of photonic Doppler velocimetry (PDV) to measure the presence of low density (<order $1 \mathrm{e} 20$ electrons/cc) plasmas via a time varying path-averaged index of refraction change $[4,5,13]$. Experiment Z3339 was an exact power flow repeat of Z3260 with the addition of the D-hole plasma detector. The D-hole plasma detector has been deployed on several DMP experiments to date, with the results of Z3339 showing the largest response by far. The PDV spectrogram is shown in Fig. 8, with red representing high signal intensity corresponding to a given PDV inferred velocity. PDV natively infers an apparent velocity, however when plasma is propagating perpendicular to the laser beam path the most appropriate interpretation of the PDV velocity signal is as a change in index of refraction of the plasma; this is the mode in which the PDV plasma detector is typically interpreted. Under this interpretation, and given the configuration of the PDV system, positive velocity represents the appearance of a plasma, while negative velocity indicates radiation impacting the fibers [5]. This interpretation is not consistent with the velocity signal shown in Fig. 8, as the observed negative velocity is inconsistent with what is known of the $Z$ machine radiation environment during this type of experiment. In experiments that utilize PDV for conventional velocity measurements we routinely observe reflections from plasma surfaces if said plasma is dense enough (see the discussion in Sec. II of Ref. [22]). We now believe that the signal observed on Z3339 is best interpreted as actual velocity from a moving plasma surface.

The first signal appears on the PDV plasma detector at $2600 \mathrm{~ns}$, which is within $10 \mathrm{~ns}$ of the prediction for onset of flow current according to the circuit model (see Fig. 5). There are then two distinct signal drop-outs until $2720 \mathrm{~ns}$ at which point the signal recovers and begins an oscillation with a negative drift throughout the remainder of the experiment. Notably the flow current exceeds $60 \mathrm{kA}$ at that time, spiking to over $140 \mathrm{kA}$ of electron current. We believe that the signal apparent at $2600 \mathrm{~ns}$ is the arrival at the probe location of free electron flow current consistent with the circuit model prediction. The signal dropouts may be due to the plasma losing reflectivity and becoming opaque-note there is a decrease in predicted flow current between $2650 \mathrm{~ns}$ and $2690 \mathrm{~ns}$ when the signal briefly recovers. Signal recovery is likely due to the plasma becoming reflective again as the density decreases.

Given our previous sensitivity studies of load current inferences we believe that the residuals evident in Fig. 7 could result in a few hundred kA of uncertainty after the onset of the loss mechanism [22]. The exact value of the PF load current after loss onset is thus in question, but none of our key assertions of catastrophic loss are affected.

\section{CONCLUSIONS}

We have presented experimental data identifying an asynchronous pulsed shaped $Z$ machine target that exhibits a catastrophic, yet repeatable, loss mechanism. Thus far, power flow diagnostics and models have focused on synchronous short pulse (100 ns rise time) drive as the simplest practical $Z$ machine drive. As these techniques mature they will have to address asynchronous pulse shaped targets with rise times of 300-1500 ns not only for application to dynamic materials program but for model validation. We have demonstrated that existing state-ofthe-art loss models fall short of accurate loss prediction for this load. The authors believe that the target presented here is an excellent candidate for initial exploration of asynchronous pulse shaped loads. Development of these models is complex, and we believe there is much room for collaboration on relevant fundamental physical concepts. 


\section{ACKNOWLEDGMENTS}

This paper describes objective technical results and analysis. Any subjective views or opinions that might be expressed in the paper do not necessarily represent the views of the U.S. Department of Energy or the United States Government. This work was conducted under the Sandia Z Fundamental Science Program. Sandia National Laboratories is a multimission laboratory managed and operated by National Technology and Engineering Solutions of Sandia LLC, a wholly owned subsidiary of Honeywell International Inc. for the U.S. Department of Energy's National Nuclear Security Administration under Contract No. DE-NA0003525.

[1] G. Laity, C. Aragon, D. Dolan, R. Falcon, M. Gomez, M. Hess, B. Hutsel, C. Jennings, M. Johnston, D. Lamppa, S. Patel, A. Porwitzky, P. VanDevender, T. Webb, G. Rochau, W. Stygar, and M. Cuneo, in 2017 IEEE Pulsed Power Conference, Brighton, United Kingdom, 2017.

[2] G. Laity et al., in 7th Euro-Asian Pulsed Power Conference \& 22nd Conference on High Power Particle Beams, Changsha, China, 2018.

[3] M. R. Gomez, R. M. Gilgenbach, M.E. Cuneo, C. A. Jennings, R. D. McBride, E. M. Waisman, B. T. Hutsel, W. A. Stygar, D. V. Rose, and Y. Maron, Experimental study of current loss and plasma formation in the $Z$ machine post-hole convolute, Phys. Rev. Accel. Beams 20, 010401 (2017).

[4] D. H. Dolan, K. Bell, B. Fox, S. C. Jones, P. Knapp, M. R. Gomez, M. Martin, A. Porwitzky, and G. Laity, Plasma and radiation detection via fiber interferometry, J. Appl. Phys. 123, 034502 (2018).

[5] A. Porwitzky, D. H. Dolan, M. R. Martin, G. Laity, R. W. Lemke, and T.R. Mattsson, Direct measurements of anode/cathode gap plasma in cylindrically imploding loads on the $Z$ machine, Phys. Plasmas 25, 063110 (2018).

[6] D. B. Seidel, W. L. Langston, R. S. Coats, M. D. Knudson, R. W. Lemke, J. Davis, and T. D. Pointon, An optimization study of stripline loads for isentropic compression experiments, in 2009 IEEE Pulsed Power Conference (2009), pp. 1165-1170, https://doi.org/10.1109/PPC.2009.5386320.

[7] R. Lemke, M. Knudson, and J.-P. Davis, Magnetically driven hyper-velocity launch capability at the Sandia Z accelerator, Int. J. Impact Eng. 38, 480 (2011), hypervelocity Impact selected papers from the 2010 Symposium.

[8] C. T. Seagle, J.-P. Davis, M. R. Martin, and H. L. Hanshaw, Shock-ramp compression: Ramp compression of shockmelted tin, Appl. Phys. Lett. 102, 244104 (2013).

[9] J.-P. Davis, J. L. Brown, M. D. Knudson, and R. W. Lemke, Analysis of shockless dynamic compression data on solids to multi-megabar pressures: Application to tantalum, J. Appl. Phys. 116, 204903 (2014).

[10] S. Root, L. Shulenburger, R. W. Lemke, D. H. Dolan, T. R. Mattsson, and M. P. Desjarlais, Shock Response and Phase
Transitions of $\mathrm{MgO}$ at Planetary Impact Conditions, Phys. Rev. Lett. 115, 198501 (2015).

[11] C. T. Seagle and A. J. Porwitzky, Shock-ramp compression of tin near the melt line, AIP Conf. Proc. 1979, 040005 (2018).

[12] L. Barker and R. Hollenbach, Laser interferometer for measuring high velocities of any reflecting surface, J. Appl. Phys. 43, 4669 (1972).

[13] O. T. Strand, D. R. Goosman, C. Martinez, T. L. Whitworth, and W.W. Kuhlow, Compact system for high-speed velocimetry using heterodyne techniques, Rev. Sci. Instrum. 77, 083108 (2006).

[14] D. H. Dolan, Accuracy and precision in photonic Doppler velocimetry, Rev. Sci. Instrum. 81, 053905 (2010).

[15] J. H. Nguyen and N.C. Holmes, Melting of iron at the physical conditions of the Earth's core, Nature (London) 427, 339 (2004).

[16] D. Valencia, R. J. O'Connell, and D. D. Sasselov, The role of high-pressure experiments on determining super-Earth properties, Astrophys. Space Sci. 322, 135 (2009).

[17] F. Wagner, F. Sohl, H. Hussmann, M. Grott, and H. Rauer, Interior structure models of solid exoplanets using material laws in the infinite pressure limit, Icarus 214, 366 (2011).

[18] A. J. Porwitzky, C. T. Seagle, and B. J. Jensen, Zero to $1,600 \mathrm{~m} / \mathrm{s}$ in 40 microns: Sensitive pulse shaping for materials characterization on Z, Procedia Eng. 204, 337 (2017), 14th Hypervelocity Impact Symposium 2017, HVIS2017, 24-28 April 2017, Canterbury, Kent, UK.

[19] B. T. Hutsel, P. A. Corcoran, M. E. Cuneo, M. R. Gomez, M. H. Hess, D. D. Hinshelwood, C. A. Jennings, G. R. Laity, D. C. Lamppa, R. D. McBride, J. K. Moore, A. Myers, D. V. Rose, S. A. Slutz, W. A. Stygar, E. M. Waisman, D. R. Welch, and B. A. Whitney, Transmission-line-circuit model of an 85-TW, 25-MA pulsed-power accelerator, Phys. Rev. Accel. Beams 21, 030401 (2018).

[20] D. J. Griffiths, Introduction to Electrodynamics, 4th ed. (Pearson, Boston, MA, 2013) re-published by Cambridge University Press in 2017.

[21] A. Robinson et al., ALEGRA: An arbitrary lagrangianeulerian multimaterial, multiphysics code, in 46th AIAA Aerospace Sciences Meeting and Exhibit, Aerospace Sciences Meetings (2008), https://doi.org/10.2514/6.2008 -1235 .

[22] A. Porwitzky and J. Brown, Uncertainties in cylindrical anode current inferences on pulsed power drivers, Phys. Plasmas 25, 063102 (2018).

[23] T. C. Wagoner et al., Differential-output $B$-dot and $D$-dot monitors for current and voltage measurements on a 20-MA, 3-MV pulsed-power accelerator, Phys. Rev. Accel. Beams 11, 100401 (2008).

[24] J. M. D. Lane, K. Leung, A.P. Thompson, and M.E. Cuneo, Water desorption from rapidly-heated metal oxide surfaces-first principles, molecular dynamics, and the Temkin isotherm, J. Phys. Condens. Matter 30, 465002 (2018). 